\title{
Randomised controlled trial of preoperative information to improve satisfaction with cataract surgery
}

\section{K Pager}

Br J Ophthalmol 2005;89:10-13. doi: 10.1136/bjo.2004.048637

Aim: To investigate the effects of an informational video on patient expectations and satisfaction with day-stay cataract surgery.

Methods: 141 patients undergoing day-stay cataract surgery were randomised into one of two video groups, explaining either what to expect from the cataract surgery or the anatomy of cataract. Patients were surveyed as to their expectations for visual outcome, anxiety, risk, and discomfort resulting from the surgery. After the operation, patients were again asked to rate their experience of anxiety, discomfort, risk, comprehension, overall satisfaction, and comparison with expectations.

Results: $84 \%$ of patients already thought they received enough or too much information. The expectations video group expected more risk and discomfort than the anatomy video group. Yet, after the surgery, the expectations video group was significantly more satisfied, understood better what was happening to them, and felt less anxious. There was no difference in the discomfort or risk actually experienced, or the expected visual outcomes. Patients with previous cataract surgery experience expected less anxiety and discomfort, and found the surgery closer to their expectations. None the less, previous cataract surgery experience did not negate the effects of the video.

Conclusions: This study demonstrates that a simple, inexpensive videotape showing patients what to expect from cataract surgery results in significant increases in patient understanding of and satisfaction with the cataract surgery, as well as a decrease in anxiety. These effects were independent of patients' expected outcomes or previous experience with cataract surgery, and despite the fact that patients generally thought they had already received enough information.

$\mathrm{P}$ atient satisfaction is an important clinical outcome. ${ }^{12}$ Not only are satisfied patients more likely to maintain a relationship with their provider, abstain from doctor shopping, refrain from malpractice litigation, and recommend the provider's services to others, ${ }^{3-5}$ but a satisfied patient is more likely to comply with treatment regimens, attend follow up appointments, recall medical advice, and go on to enjoy improved health outcomes. ${ }^{3}$

One of the most important determinants of patient satisfaction is information. ${ }^{67}$ Studies in other medical fields have found that preoperative education is associated with less anxiety, fewer complications, shorter hospital stays, less pain relief medication, and increased satisfaction. ${ }^{6}{ }^{89}$ Lack of information is one of the most common sources of patient dissatisfaction, ${ }^{710}$ and studies have consistently shown that patients want more information than they received, ${ }^{7}$ and believe they received less information than their surgeons believed they were providing. ${ }^{5}$
Although cataract surgery is one of the most frequent and privatised surgical procedures worldwide, ${ }^{11}$ little research has addressed the role of preoperative information and satisfaction in the context of cataract surgery. One study found that, unlike patients in other medical fields, $26 \%$ of cataract patients explicitly said they did not wish to receive preoperative information, and $28 \%$ found such information anxiety provoking. ${ }^{12}$ This is in spite of findings that $93 \%$ of patients are unable to relay even basic information concerning the procedure or its complications before surgery, ${ }^{12}$ that only $4 \%$ of patients remember more than two risks of surgery even one day after the operation, ${ }^{13}$ and that previous studies have found between $5 \%$ and $25 \%$ of patients are dissatisfied with their cataract surgery. ${ }^{14}{ }^{15}$ Preoperative education is essential not only to patient satisfaction but also for informed consent.

It is widely accepted that patient satisfaction is largely mediated through patient expectations, where meeting or exceeding patient expectations results in satisfaction. ${ }^{16}{ }^{17}$ There has been little research into this effect concerning cataract surgery, but it is important to consider whether any increase in patient satisfaction achieved through preoperative education operates by altering patient expectations.

The purpose of this study is to use a randomised controlled trial to investigate whether a preoperative educational videotape increases patient satisfaction with day-stay cataract surgery, and whether this effect is mediated through reducing patient expectations.

\section{METHODS \\ Patients}

In all, 160 consecutive patients undergoing day-stay cataract surgery at Sydney Private Hospital were invited to participate in this study when first registering at the hospital. Sydney Private Hospital is the largest private provider of cataract surgery in New South Wales, and serves a large cross section of patients throughout metropolitan Sydney. Virtually all day-stay cataract procedures involved regional anaesthesia and extracapsular extraction using phacoemulsification.

\section{Intervention}

Patients were shown one of two possible 9 minute videotapes. Both videotapes were of similar length and style, professionally produced and edited, and complied from patient education videos produced by pharmaceutical companies, with occasional voiceover added. The "expectations" video described the experience of undergoing cataract surgery, as well as mention of the potential risks. One patient was followed from arrival at the hospital through to discharge, including registration, donning a hospital gown, instillation of eye drops, transfer to the operation theatre, anaesthetics, drapes, and a short view of the surgery itself. The purpose of this video was to convey what sensations the patient is likely to experience during his or her half day at the hospital. To control for any satisfaction benefits merely from 


\begin{tabular}{|lllll|}
\hline Table 1 Patient characteristics & & & \\
\hline Characteristic & All patients & $\begin{array}{l}\text { Expectations } \\
\text { video }\end{array}$ & $\begin{array}{l}\text { Anatomy } \\
\text { video }\end{array}$ & Significance \\
\hline Total number & 141 & 73 & 68 & \\
Mean age (SD) & $73.8(9.2)$ & $74.6(9.0)$ & $72.9(9.4)$ & NS \\
No of females (\%) & $91(64.5 \%)$ & $49(64.5 \%)$ & $42(64.5 \%)$ & NS \\
Median preoperative VA: 6/(1 st, 3rd & $18(12,36)$ & $18(12,36)$ & $18(12,36)$ & NS \\
quartile) & $84.7(14.8)$ & $83.4(16.4)$ & $86.0(13.0)$ & NS \\
Mean preoperative VF-14 (SD) & $55(39.0 \%)$ & $28(38.4 \%)$ & $27(39.7 \%)$ & NS \\
No of previous cataract (\%) & $6(6,9)$ & $6(6,9)$ & $6(6,9)$ & NS \\
Median VA at 1 month: 6/(1 st, 3rd & $89.8(16.4)$ & $88.5(17.9)$ & $91.2(14.5)$ & NS \\
quartile) & & & & \\
Mean VF-14 at 1 month (SD) & & & & \\
\hline VA, visual acuity. & & & \\
Test: Mann-Whitney U for visual acuity, two tailed independent $T$ tests for others. & \\
\hline
\end{tabular}

being involved with the study (Hawthorne effect), the control group also watched a placebo or "anatomy" video which contained no information at all about what patients should expect from cataract surgery, but instead was exclusively concerned with the anatomy of the crystalline lens and the causes and nature of cataracts. This video was designed to be similar in style, interest, and relevance to patients but addressed the question "how did I get this cataract?" rather than the procedure for removing it. Institutional ethics approval was obtained for the use of these videos and the data collection described below.

\section{Procedure and measures}

After obtaining informed consent, the patient's age, sex, previous experience with cataract surgery, and current visual function were recorded by a research interviewer, along with a question about the amount of information the patient had already received regarding this surgery. Visual function was measured using the VF-14, ${ }^{18}$ a widely used scale based on difficulty conducting common activities, with final scores ranging from 0 (no visual ability) to 100 (no visual disability). The patient was then randomised into the expectation or anatomy group, and the appropriate videotape was shown to the patient. The videotape was labelled only with $\mathrm{A}$ or $\mathrm{B}$, and the interviewer was masked to the patient's videotape allocation. After the completion of the videotape, patients were asked to rate their satisfaction with current vision and their expectations for the upcoming cataract surgery with regard to anxiety, discomfort, and risk. Answers to all of these questions were recorded on a 12 centimetre visual analogue scale labelled "not at all" and "extremely" at either end. The validity and reliability of visual analogue scales have been established previously. ${ }^{19}$ Finally, the VF-14 questions were posed again, but for each item the patient was asked how much trouble he or she expected after the surgery. An "expected VF-14" score was recorded.

Before discharge, the patient was asked to rate his or her experience of the cataract surgery in terms of anxiety, discomfort, and risk using identical visual analogue scales. In addition, patients were also asked about how the experience compared with what they were expecting, their

$\begin{aligned} & \text { Table } 2 \\
& \text { patients (all combined) }\end{aligned}$
\begin{tabular}{ll}
\hline Information supplied & Number (\%) \\
\hline No information & $2(1.4)$ \\
Not enough & $19(13.5)$ \\
Enough & $115(81.6)$ \\
Too much & $4(2.8)$ \\
\hline Total: $99.3 \%, n=140$ with one missing value.
\end{tabular}

level of understanding, and overall satisfaction. Answers to these questions were also recorded on visual analogue scales, the first labelled "about the same" at the midpoint and "much worse" or "much better" at either end. The others were labelled, as before, with "not at all" and "extremely" at each end. The exact phrasing of the questions posed is included in the appendix.

To control for possible differences in clinical outcome between groups, VF-14 visual disability and operative eye Snellen visual acuity were recorded before surgery at 1 month follow up.

\section{Data analysis}

The visual analogue scale markings were converted into a scale of 0 to 10, and all data were double entered and verified using an Excel spreadsheet, then converted into SPSS for Windows version 11 for further analysis. The sample was based on a requirement of 120 patients to detect an effect size of 0.5 with a power of $80 \%$ assuming a $75 \%$ participation rate.

Non-parametric analysis using the Mann-Whitney U test was employed to compare visual analogue scale results between the expectations and anatomy video groups. For other comparisons, such as age, two tailed independent $T$ tests were employed. Finally, possible interactions between the effects of video allocation and previous cataract surgery were measured using an ordered logistic regression, entering video allocation, previous cataract surgery and their interaction as independent variables.

\section{RESULTS}

A total of 160 patients were approached and 141 (88\%) agreed to participate. Basic preoperative characteristics of these patients are presented in table 1 . The average age was 74 (SD 9) years, 65\% were female, and 39\% had previous cataract surgery. There were no significant differences between video groups for any demographic or clinical variable recorded, confirming effective randomisation.

Table 2 presents all patients' responses when asked (before viewing any video) about the amount of information they had already received concerning what to expect from the cataract surgery. Eighty four per cent of patients thought they had received enough or too much information, and only $15 \%$ thought they received not enough or no information at all.

Patients who had viewed the expectations video were more likely to think the surgery would be uncomfortable $(2.5 \vee 1.6$, $\mathrm{p}=0.008)$ or risky $(2.5 \vee 1.9, \mathrm{p}=0.009)$. However, video allocation had no effect on patient anxiety, satisfaction with current vision, or expected postoperative VF-14. Further information on the effects of video allocation on patients' preoperative expectations is presented in table 3.

When surveyed after the surgery, patients who had been allocated to the expectations video were significantly more 
Table 3 Patients' mean (SD) preoperative expectations

\begin{tabular}{llll}
\hline Expectation & $\begin{array}{l}\text { Information } \\
(\mathbf{n}=\mathbf{7 3})\end{array}$ & $\begin{array}{l}\text { Anatomy } \\
(\mathbf{n}=68)\end{array}$ & Significance \\
\hline Nervous or uneasy & $6.67(2.3)$ & $7.16(2.2)$ & $\mathrm{NS}$ \\
Uncomfortable or painful & $2.48(1.8)$ & $1.60(1.2)$ & $\mathrm{p}=0.008$ \\
Dangerous or risky & $2.50(1.8)$ & $1.89(1.7)$ & $\mathrm{p}=0.009$ \\
Current satisfaction with vision & $3.79(2.1)$ & $3.41(2.0)$ & $\mathrm{NS}$ \\
Expected postoperative VF-14 & $83.42(16.4)$ & $86.03(13.0)$ & $\mathrm{NS}$ \\
\hline Test: Mann-Whitney U. VF-14 scale is 0 to 100 (no disability; other scales: 0=not at all, 10=extremely).
\end{tabular}

likely to think they understood what was happening to them (7.4 $v$ 5.8, $\mathrm{p}<0.001)$ and be satisfied with the overall experience $(8.2 \vee 7.8, \mathrm{p}=0.036)$. In addition, they were less likely to report having felt nervous or uneasy during the surgery $(0.9 v 1.3, \mathrm{p}<0.001)$. Further information on the effects of video allocation on patients' postoperative impressions is presented in table 4 .

Table 5 compares the preoperative expectations and postoperative impressions of patients who had or had not experienced cataract surgery previously. Not surprisingly, patients who had previously experienced cataract surgery were more satisfied with their preoperative vision $(2.2 \vee 4.1$, $\mathrm{p}=0.0183)$, expected to be less nervous (2.5 $v$ 3.5, $\mathrm{p}=0.0015)$, and expected the surgery to be less uncomfortable $(1.4 v 2.5, \mathrm{p}<0.0001)$ than did patients who had not previously experienced cataract surgery. After surgery, patients who had previous experience were more likely to find the cataract surgery close to what they had expected (6.4 $v 7.9, \mathrm{p}<0.0001)$, but otherwise had identical impressions regarding anxiety, risk, discomfort, understanding, and general satisfaction. Finally, an ordered logistic regression (not shown) examined the interaction effect of video allocation and previous cataract experience, controlling for each factor individually. Across all five preoperative variables and six postoperative variables, there were no interaction effects: the significant effects of video allocation operated independently of patients' previous experience with cataract surgery.

\section{DISCUSSION}

This study has demonstrated that a simple, inexpensive videotape showing patients what to expect from cataract surgery resulted in significant increases in patient understanding of and satisfaction with cataract surgery, as well as a decrease in reported anxiety. These effects were independent of patients' previous experience with cataract surgery, and despite the fact that - as with previous studies ${ }^{12}$ - these patients generally thought they had already received enough information about what to expect from the surgery.

This is only the second controlled study to assess the role of preoperative information in cataract surgery, the previous study dating back to 1982 and using an informational audiotape, yielding no significant effects. ${ }^{20}$ Naturally, cataract surgery has advanced considerably since 1982, and might now be considered a different procedure entirely. The findings of the present study are important owing to the significant impact increased patient satisfaction has upon health outcomes, combined with the marketing, financial, and litigation benefits satisfied patients bestow upon their service providers. ${ }^{3-5}{ }^{17}$ Despite its potential benefits, cataract patients did not seek out and may even avoid additional information, which presents additional concerns regarding informed consent, considering the very low level of understanding possessed by many patients undergoing cataract surgery. ${ }^{12} 13$

The extent to which most patients were highly anxious preoperatively and found the surgery to exceed their expectations demonstrates that there is room for improvement in allaying patient fears and better aligning their expectations for surgery with the actual experience.

The video allocation had no effect on patients' preoperative anxiety or expected visual outcomes. However, it did increase expected discomfort and risk. This may have resulted from close up footage of actual cataract surgery, as well as a clear discussion of potential risks, both presented only to the expectations video group. Previous research has suggested that information too directly related to the actual procedure may increase anxiety, and attention is best directed to factors peripheral to the actual surgery, such as the drapes over the patient's head and the need to lie still. ${ }^{12}$ Further research is required to tailor informational presentations to best allay patient anxiety, promote satisfaction and fulfil the need for adequate informed consent. Additionally, this study is limited to satisfaction with the day-stay surgery experience, and does not address the follow up care of longer term effects.

This study did not support the hypothesis explaining patient satisfaction in terms of expectations. Patients in the expectations video group neither expected lower outcomes nor found the actual surgery to exceed their expectations by a larger margin than did the control group of patients watching the anatomy video. Although no more anxious than the anatomy video group, the expectations video patients did expect more discomfort and risk, and the increase in postoperative satisfaction could also be the result of relief that the surgery was not as risky or uncomfortable as they had feared. However, if this were a dominant factor we would have expected increased preoperative anxiety and a

Table 4 Patients' mean (SD) postoperative impressions

\begin{tabular}{llll}
\hline Impression & $\begin{array}{c}\text { Information } \\
(\mathbf{n}=\mathbf{7 3})\end{array}$ & $\begin{array}{c}\text { Anatomy } \\
(\mathbf{n}=68)\end{array}$ & Significance \\
\hline Comparison with expectation & $7.45(1.5)$ & $7.21(1.7)$ & $\mathrm{NS}$ \\
Nervous or uneasy & $0.88(0.9)$ & $1.29(1.0)$ & $\mathrm{p}<0.001$ \\
Uncomfortable or painful & $0.80(0.8)$ & $0.95(0.9)$ & $\mathrm{NS}$ \\
Dangerous or risky & $1.54(1.4)$ & $1.27(1.2)$ & $\mathrm{NS}$ \\
Understand what's happening & $7.44(2.0)$ & $5.82(2.8)$ & $\mathrm{p}<0.001$ \\
Satisfaction with experience & $8.19(1.0)$ & $7.84(1.0)$ & $\mathrm{p}=0.036$ \\
\hline Test: Mann-Whitney U. VF-14 scale is 0 to 100 (no disability). Comparison with expected, 5=as expected, & \\
O=much worse, 10=much better; other scales: 0= not at all, 10=extremely. &
\end{tabular}


Table 5 Effect of previous cataract surgery on preoperative expectations and postoperative impressions

\begin{tabular}{llll} 
& $\begin{array}{l}\text { First time } \\
\text { surgery }\end{array}$ & $\begin{array}{l}\text { Previous } \\
\text { surgery }\end{array}$ & Significance \\
\hline Preoperative expectations & $3.49(2.1)$ & $2.48(2.4)$ & $\mathrm{p}=0.002$ \\
Nervous & $2.48(1.6)$ & $1.44(1.5)$ & $\mathrm{p}<0.001$ \\
Painful & $2.15(1.7)$ & $2.32(1.9)$ & $\mathrm{NS}$ \\
Risky & $96.31(10.5)$ & $95.68(9.9)$ & $\mathrm{NS}$ \\
Expected VF-14 & $4.07(1.9)$ & $2.15(1.7)$ & $\mathrm{p}=0.018$ \\
Current satisfaction & & & $\mathrm{p}<0.001$ \\
Postoperative impressions & $7.93(1.3)$ & $6.44(1.5)$ & $\mathrm{NS}$ \\
Compared & $1.06(0.9)$ & $1.10(1)$. & $\mathrm{NS}$ \\
Nervous & $0.90(0.8)$ & $0.83(0.9)$ & $\mathrm{NS}$ \\
Painful & $1.34(1.3)$ & $1.51(1.4)$ & $\mathrm{NS}$ \\
Risky & $6.97(2.2)$ & $6.19(2.9)$ & $\mathrm{NS}$ \\
Understand & $8.06(1.0)$ & $7.95(1.0)$ & \\
Satisfied & &
\end{tabular}

Tests: Mann-Whitney U for first versus previous surgery; VF-14 scale is 0 to 100 (100=no disability). Comparison with expected: $5=$ as expected, $0=$ much worse, $10=$ much better. Other scales: $0=$ not at all, $10=$ extremely.

larger margin of exceeded expectations postoperatively, which we did not.

This study shows that an intervention such as a videotape explaining to patients the sensations they are likely to experience during surgery, along with common outcomes and risks, reduces anxiety and improves patient satisfaction following cataract surgery. This simple intervention has benefits for the patient, surgeons, and the community.

\section{ACKNOWLEDGEMENTS}

The author would like to thank Allergan and Alcon for permission to use their patient education videotapes and brochures concerning cataract surgery.

This study was supported in part by the National Health and Medical Research Council of Australia.

Correspondence to: Dr Chet K Pager, Department of Clinical Ophthalmology and Eye Health, University of Sydney, and Royal Prince Alfred Hospital, 67 Missenden Road PO, Camperdown, NSW 2050, Australia; ckpager@stanfordalumni.org

Accepted for publication 12 July 2004

\section{REFERENCES}

1 Williams B. Patient satisfaction: a valid concept? Soc Sci Med 1994;38:509-16.

2 Epstein AM. The outcomes movement-will it get us where we want to go? N Engl J Med 1990;323:266-70.

3 Aharony L, Strasser S. Patient satisfaction: what we know about and what we still need to explore. Med Care Rev 1993:50:49-79.

4 Zeithaml VA, Parasuraman A, Berry LL. Delivering quality service: balancing customer perceptions and expectations. New York: The Free Press, 1990.

5 Angelopoulou P, Kangis P, Babis G. Private and public medicine: a comparison of quality perceptions. International Journal of Health Care Quality Assurance Incorporating Leadership in Health Services 1998;11:14-20.

6 Padberg RM, Padberg LF. Strengthening the effectiveness of patient educaiton: applied principles of adult education. ONF 1990;17:65.

7 Harris J. You can't ask if you don't know what to ask: a survey of the information needs and resources of hospital outpatients. N Z Med J 1992; 105: 199-202.

8 Hathawy D. Effects of preoperative instruction on postoperative outcomes: a meta-analysis. Nurs Res 1986;32:269-75.

9 Cupples SA. Effects of timing and reinforcement of preoperative education on knowledge and recovery of patients having coronary artery bypass graft surgery. Heart Lung 1991;20:654-60.

10 Carr-Hill RA. The measurement of patient satisfaction. J Public Health Med 1992;14:236-49.

11 Taylor HR. Cataract: how much surgery do we have to do? Br J Ophthalmol 2000;84:1-2.

12 O'Malley TP, Newmark TS, Rothman MI, et al. Emotional aspects of cataract surgery. Int J Psychiatry Med 1989;19:85-9.

13 Morgan LW, Schwab IR, Informed consent in senile cataract extraction. Arch Ophthalmol 1986;104:42-5.
14 Legro MW. Quality of life and cataracts: a review of patient-centered studies of cataract surgery outcomes. Ophthalmic Surg 1991;22:341-3.

15 Monestam E, Wachtmeister L. Dissatisfaction with cataract surgery in relation to visual results in a population-based study in Sweden. J Cataract Refract Surg 1999;25:1127-34

16 Thompson AG, Sunol R. Expectations as determinants of patient satisfaction: concepts, theory and evidence. Int J Qual Health Care 1995;7:127-41.

17 Yucelt, $U$. An investigation of causes of patient satisfaction/dissatisfaction with phyiscian services. Health Marketing Q 1994;12:11-28.

18 Steinberg EP, Tielsch JM, Schein OD, et al. The VF-14. An index of functional impairment in patients with cataract. Arch Ophthalmol 1994;1 12:630-8.

19 Scrimshaw SV, Maher C. Responsiveness of visual analogue and McGill pain scale measures. J Manip Physiol Ther $2001 ; 24: 501-4$.

20 Hill EJ. Sensory information, behavioral instructions and coping with sensory alteration surgery. Nurs Res 1982;31:17-21.

\section{APPENDIX}

\section{QUESTIONS POSED TO PATIENTS}

After the completion of the videotape, approximately 1 hour before surgery, each patient was asked about his or her expectations for the upcoming cataract surgery through the following questions:

- How nervous or uneasy do you think you will feel during the cataract surgery?

- How uncomfortable or painful do you think the cataract surgery will be?

- How dangerous or risky do you think the cataract surgery will be?

Answers to all of these questions were recorded on a 12 centimetre visual analogue scale labelled with "not at all" and "extremely" at either end.

Approximately 2 hours after the operation, just before discharge, the patient was asked to rate his or her experience of the cataract surgery along the same three dimensions listed above, using identical visual analogue scales. In addition, patients were also asked:

- How did you find the cataract surgery, compared to what you were expecting?

- How well did you understand what was happening to you?

- How satisfied were you with the whole experience of having your cataract taken out?

Answers to these questions were also recorded on visual analogue scales, the first labelled "about the same" at the midpoint and "much worse" or "much better" at either end. The others were labelled, as before, with "not at all" and "extremely" at each end. 